\title{
الفن الرقمي وتأثيره على تقديم تجارب جمالية فربدة في ألعاب الفيديو \\ Digital art and its impact on delivering unique aesthetic experiences in video games
}

مدرس بقسم الرسوم المتحركة كلية الفنون الجميلة- جامعة المنيا

أميرة عبد الوهاب احمد عبد الوهاب

مقدمة : مقير

لقد تطورت ألعاب الفيديو من تصميم بدائي إلى استغلال القدرات الترفيهية للكمبيوتر الحديث ثم إلى شكل جديد ومتطور

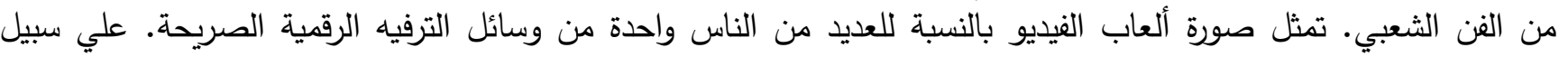

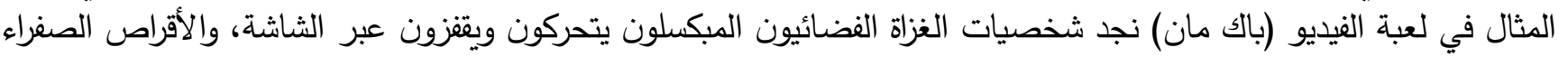

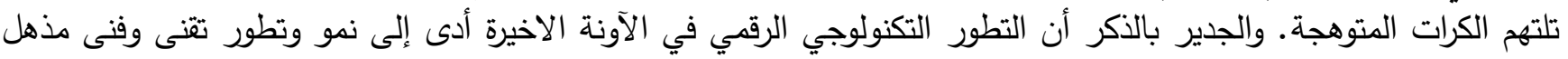

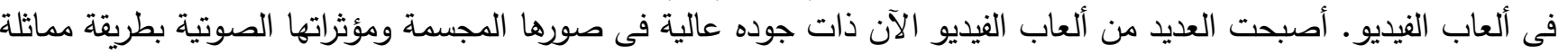

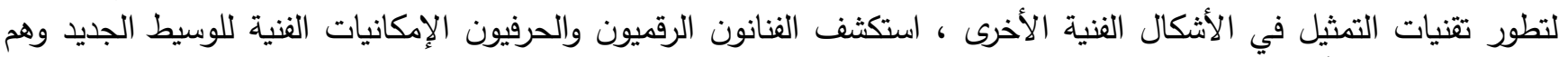

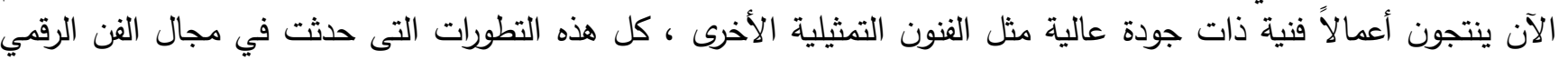
جعلت هذا التطور للشكل الثقافي الجديد تجربة مثيرة.

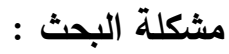

- ما دور الفن الرقمي في تصميم ألعاب الفيديو وما مدى تأثيره على جماليات الألعاب.

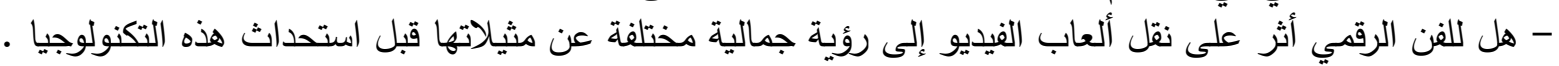
أهداف البحث :

$$
\begin{aligned}
& \text { - - - تعريف الفن الرقمي والأدوات الرقمية. }
\end{aligned}
$$

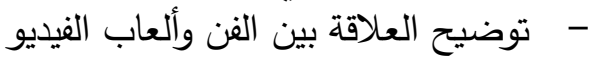

$$
\begin{aligned}
& \text { - } \\
& \text { - - - إلقاء الضوء على تأثير الفن الرقمي على جماليات بعض الألى العاب. }
\end{aligned}
$$

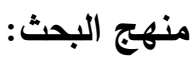

$$
\begin{aligned}
& \text { أتبعت الباحثة في هذه الدراسة المنهج التاريخي والوصفي التحليلي . } \\
& \text { حدود البحث : - (لبعت } \\
& \text { من الستينات إلى القرن الحادي والعشرون. } \\
& \text { فروض البحث: } \\
& \text { 1- أن هناك علاقة وثيقة بين الفن الرقمي وألعاب الفيديو. } \\
& \text { 2-2 أن أنماط الفن الرقمي لها دور كبير في تصميم ألعاب الفي الفيديو. } \\
& \text { 3- أن الفن الرقمي له تأثثير كبير على جلى أنماليات ألعاب الفيديو. }
\end{aligned}
$$

لا يختلف الفن الرقمي كثيراً عن الفن القديم و لكن التقدم التكنولوجي زاد من الإدراك الحسي والفهم للفن وتغطية أحداثه ،

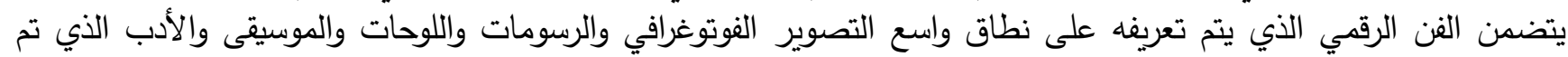

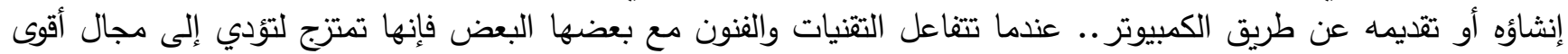

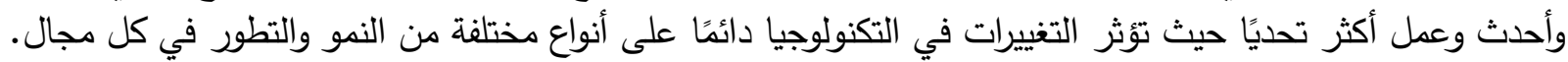

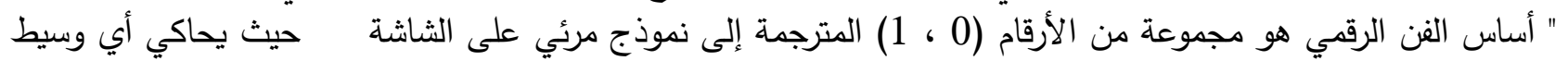

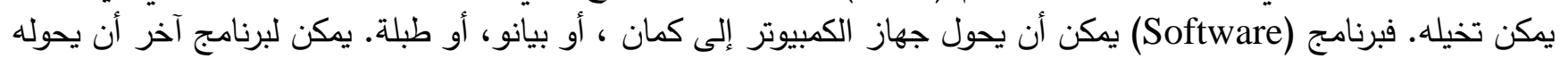

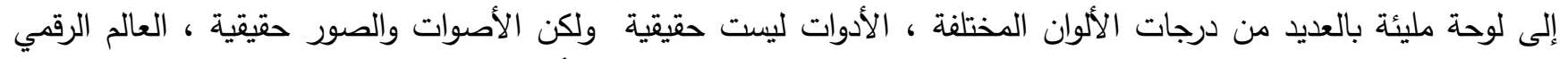

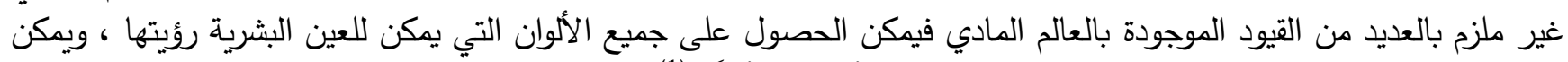

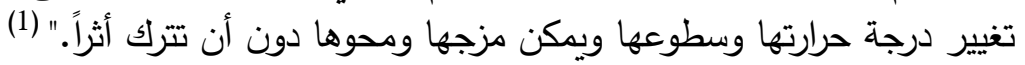

(1) Autodesk, Inc. “How Is Digital Drawing Different from Traditional Art?”November 14, 2017 https://www.sketchbook.com/blog/digital-drawing-different-traditional-art/ , 22/1/2019. 
إذا تم تحليل الأشياء الفنية عن كثب ، فيمكن وصفها في جوهرها كمحتوى معلومات ذو طبيعة إدراكية معينة (بصري ،

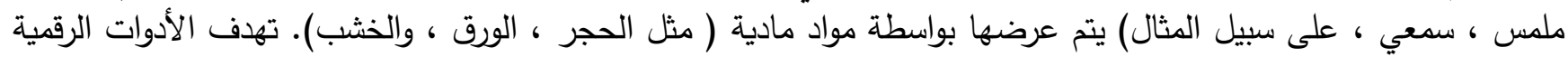

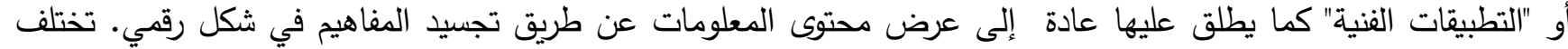

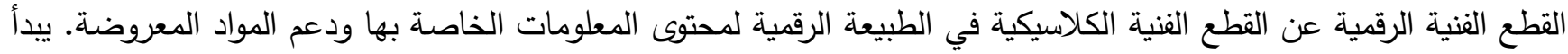

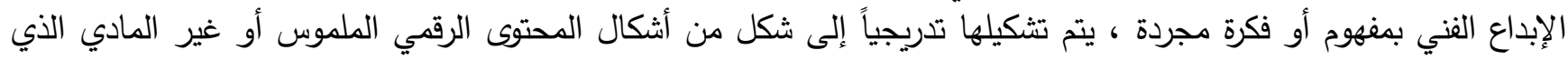

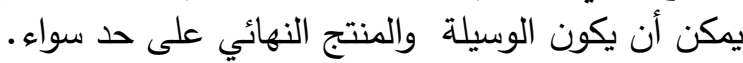

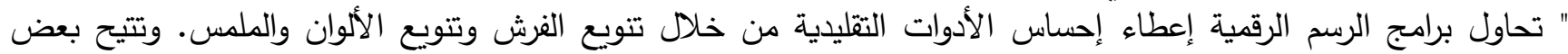

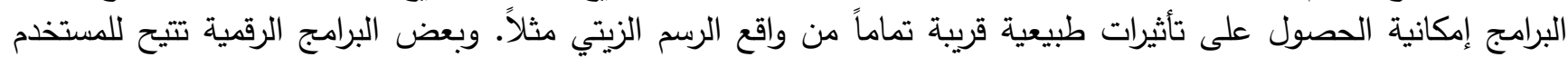

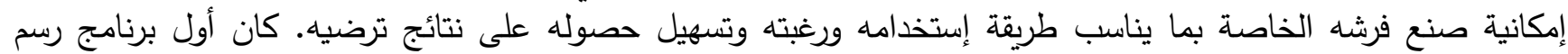

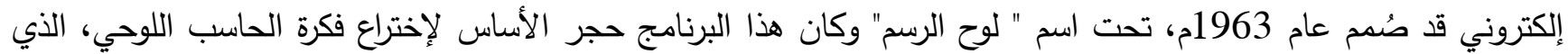

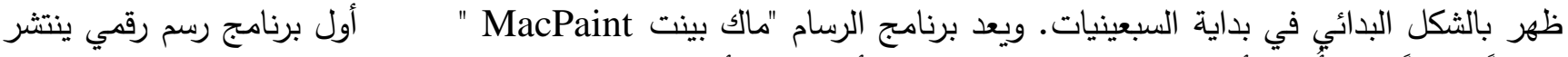

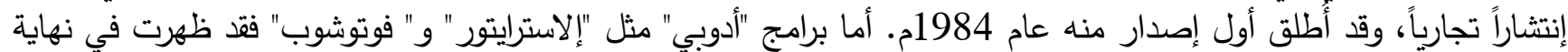

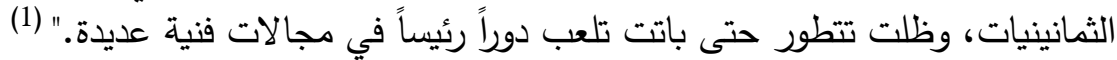

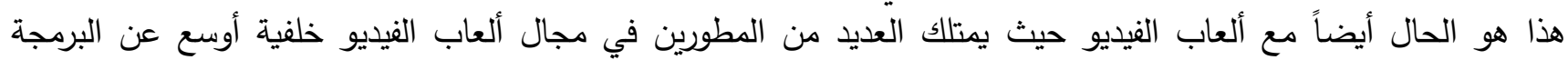
ولديهم خلفيات أيضاً في الأفلام والموسيقى والفن ويجمعون هذه الخلفيات مع خبراتهم التكنولوجية. الفن الرقمي التفاعلي

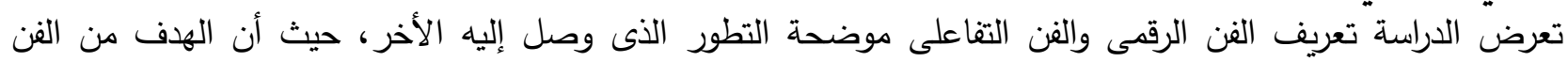

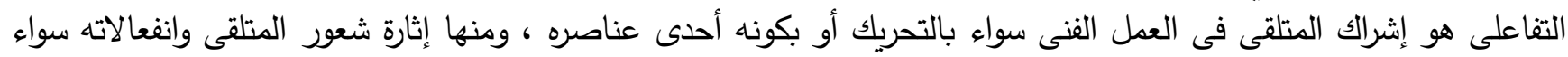

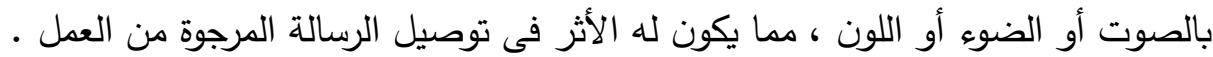

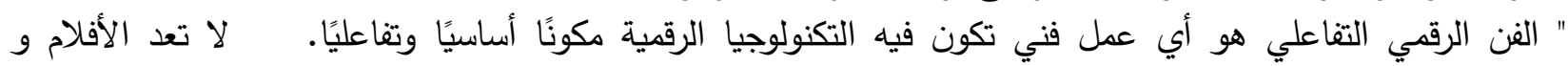

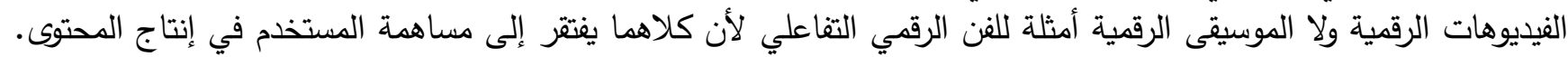

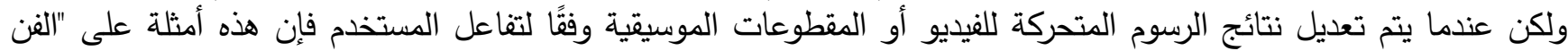

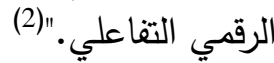

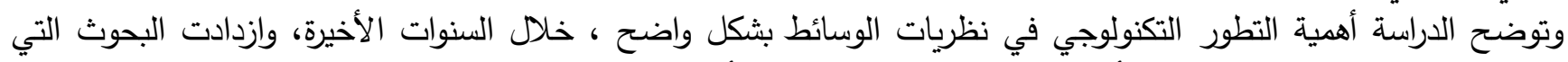

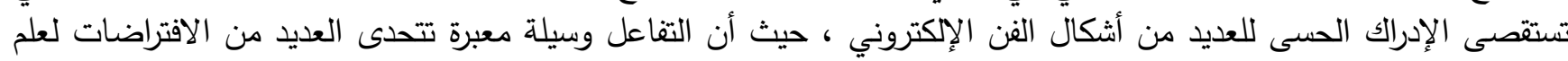

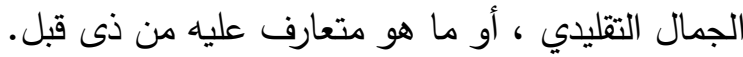
دور الفنان في تصميم ألعاب الفيديو

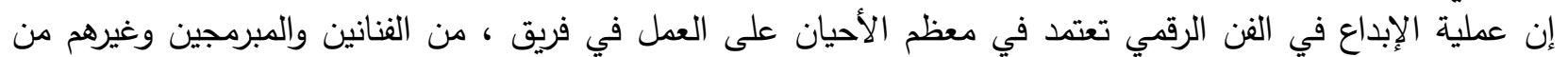

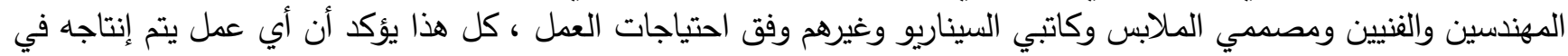

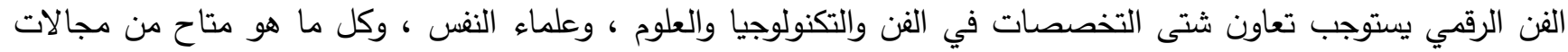

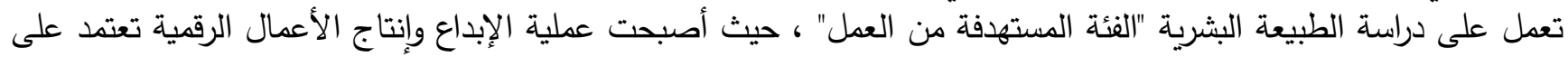
التكامل والمعالجات للمحتوى الرقمي لإنتاج أعمال فنية.

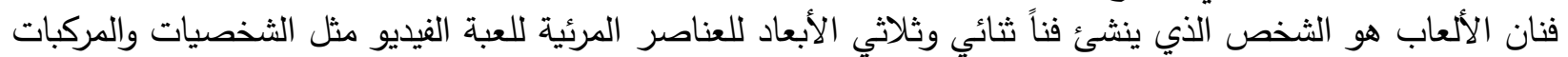

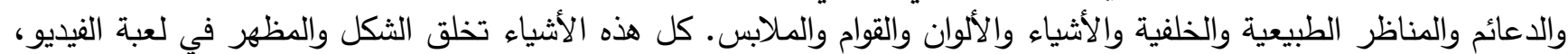

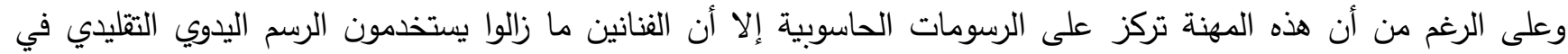

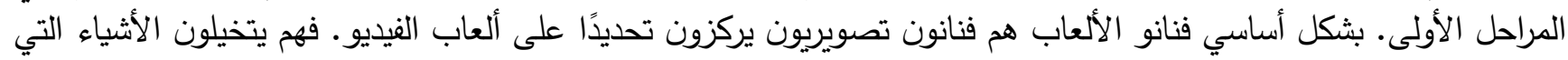

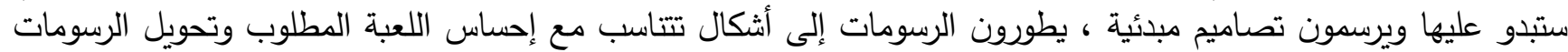
المختارة إلى رسومات حاسوبية ثنائية أو ثلاثية الأبعاد.

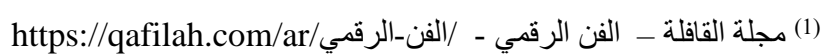

(2) Enrico Nardelli “A classification framework for interactive digital artworks” International Conference on User Centric Media UCMEDIA 2010, p. 91. 
فنانو الألعاب يخلقون الجوانب الفنية للعبة باستخدام برامج مختلفة لصنع الفن الرقمي. قد تتطلب بعض الألعاب أيضاً من الفان

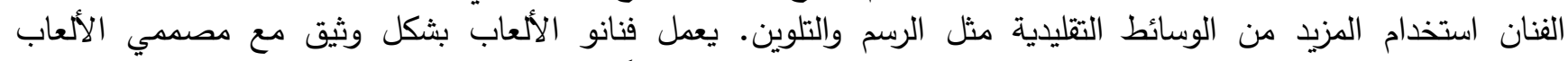
والمبرمجين وما إلى ذلك لإنشاء فن اللعبة. لذلك سوف تعرض الدراسة تصنيفاً لفنانو الألعاب وكيفية عملهم.

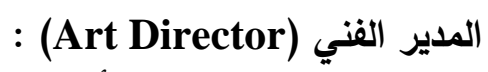
هو الذى يقوم بالتنسيق بين أقسام الفنون المختلفة ، ومصدمي اللعبة ، وهو الذى يقود الفرق الفنية للعبة والرسوم المتحركة ، وهو

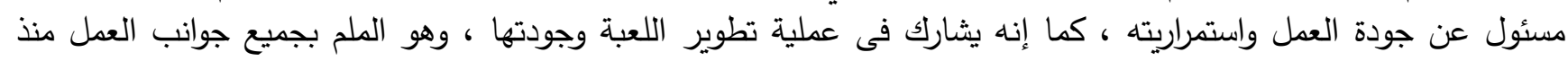
كونها فكرة إلى أن تتتج كعمل فنى الكترونى .

الفنان المفكر(Concept Artist) : عندما يصل المدير الفني إلى فكرة حول شخصية أو لعبة جديدة فإن الفنان المفكر هو

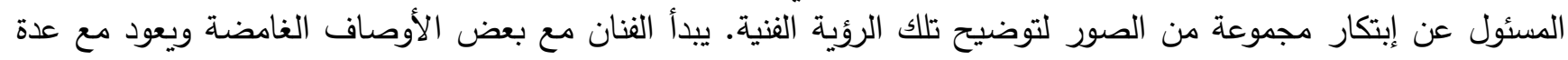

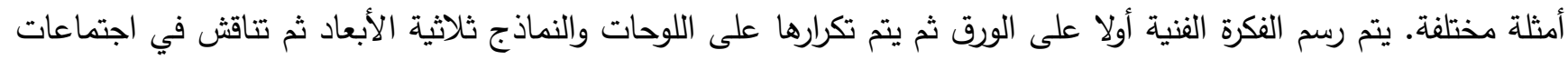

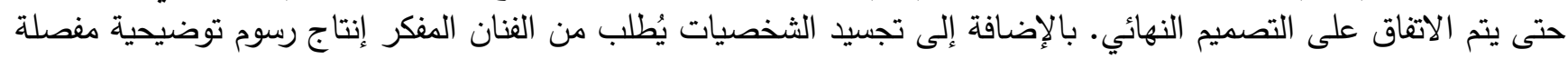

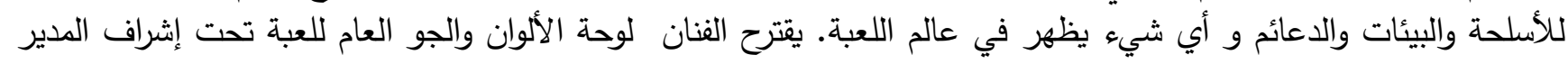

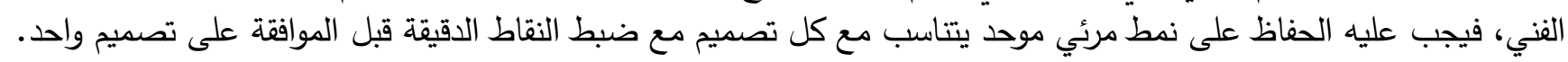
يتطلب هذا التكرار الصبر والإهتمام الثديد بالتفاصيل فودي موحئ.

فنان البيئة (Environment Artist): المعروف أيضًا باسم عارض البيئة يقوم بتصميم خلفيات ومشاهد ألعاب الفيديو

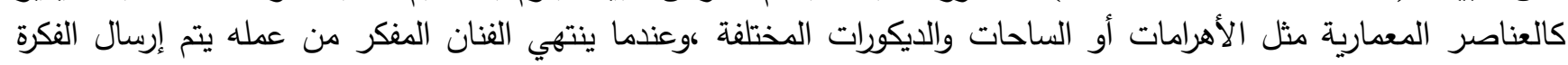

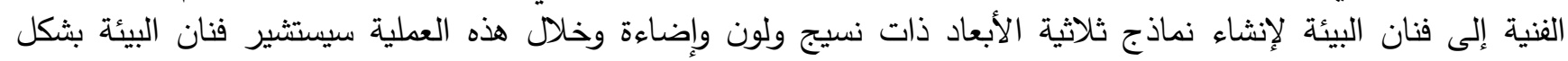

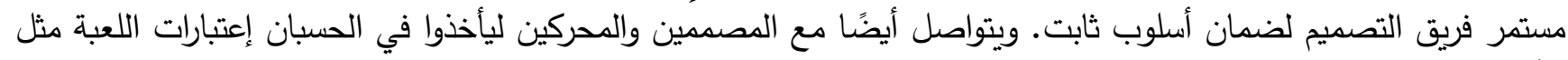

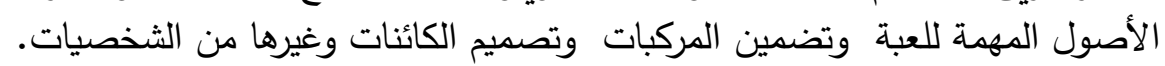

فنان الشخصية (Character Artist) : هو الفنان المتخصص في تصميم شخصيات اللعبة حيث يمكنه أن يبتكر أكثر

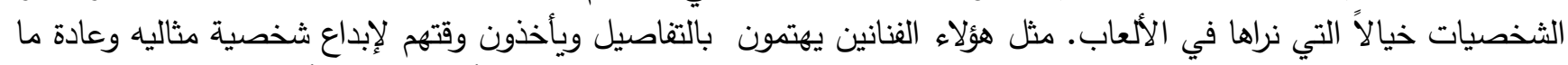

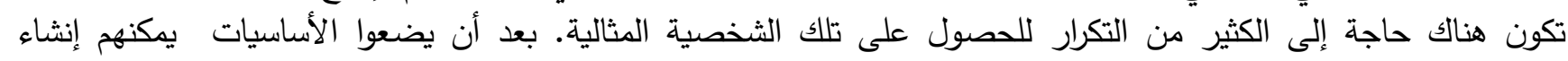

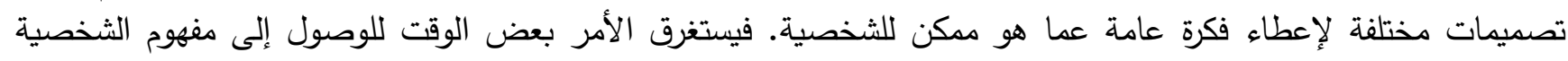
النهائي.

\section{الأنماط الفنية في ألعاب الفيديو}

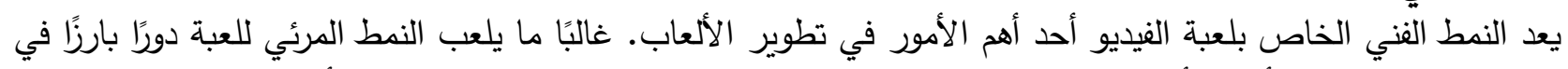

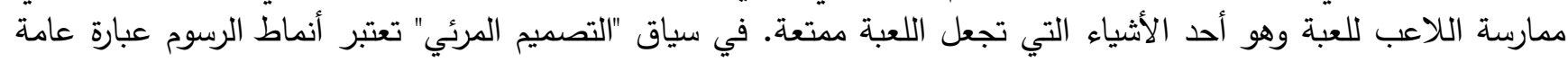

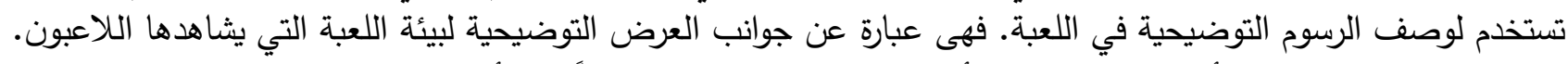

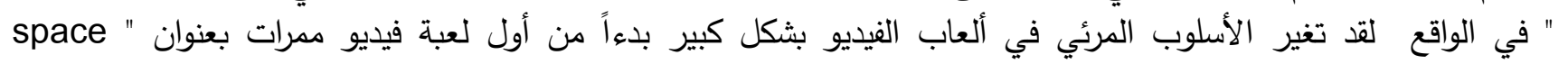
"computer

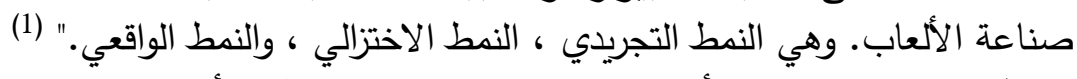

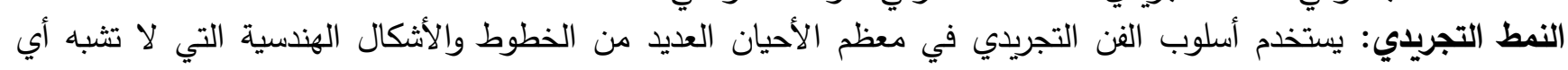

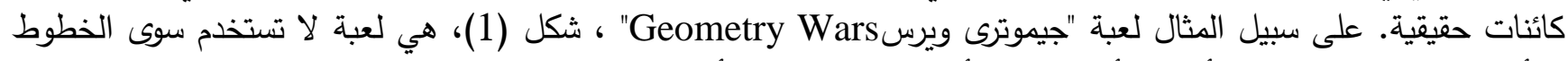

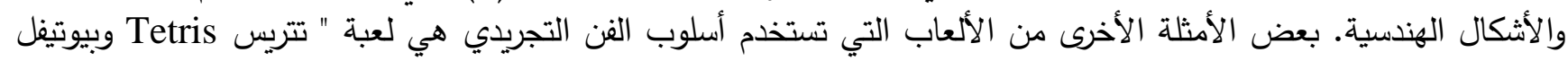

كاتامارى Beautiful Katamari

(1) Lee, L., Gee, S. \& Dolah, J. "Graphic Styles Appearance in Educa-tional Games to Enhance Malaysian Students Learning: A Preliminary Study". $2^{\text {nd }}$ international conference on creative media, design \& technology (REKA 2016), p. 2 . 


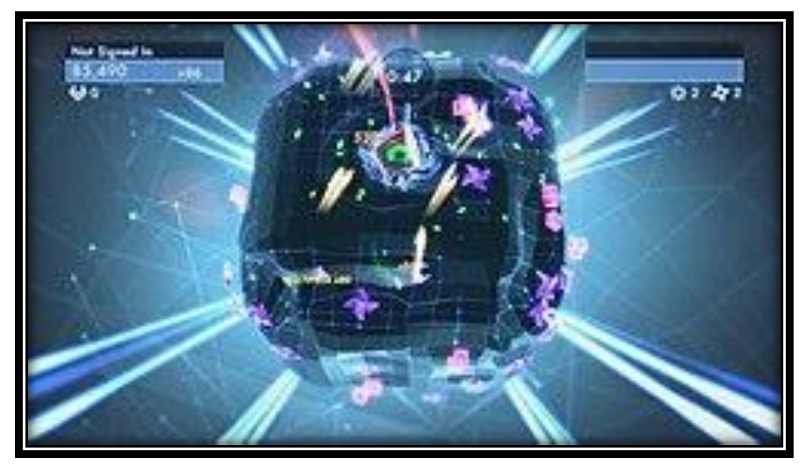

شكل (1) كادر من لعبة Geometry Wars 3: Dimensions

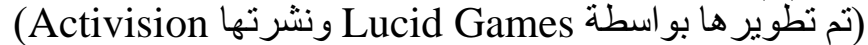

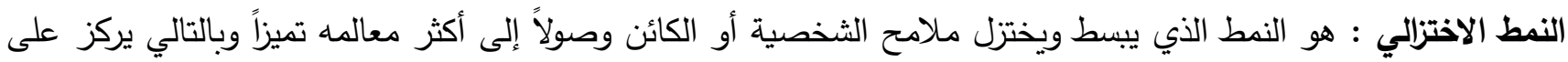

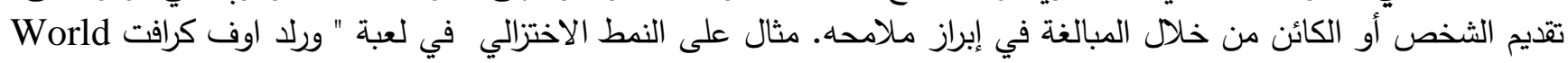

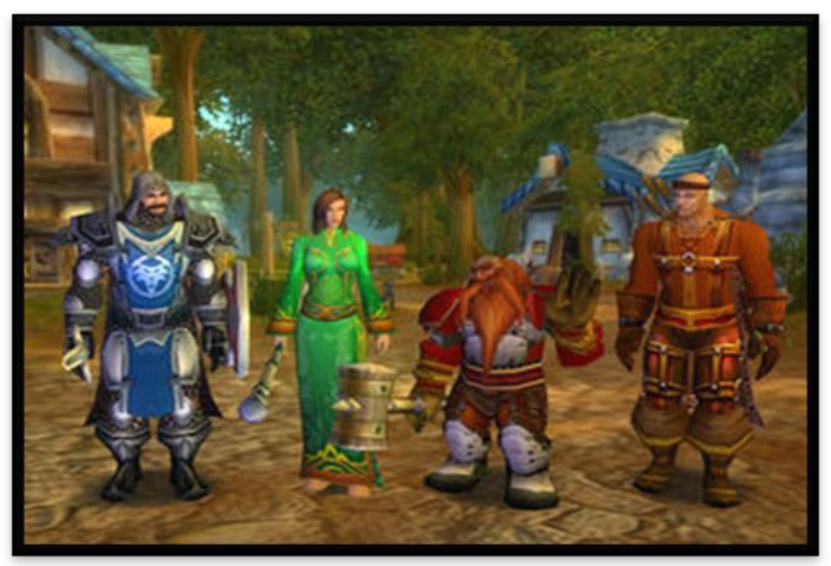

of Warcraft

النمط الواقعي: "هذا النمط من الأساليب الفنية الأكثر استخدامًا في الجيل الحالي من منصات ألعاب الفيديو. حيث أن الأشياء

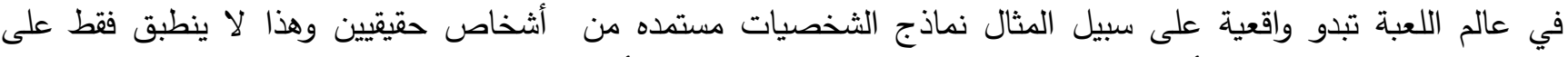

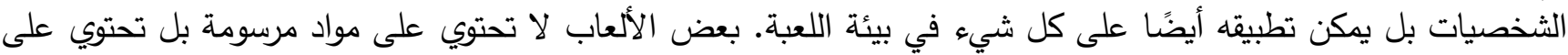

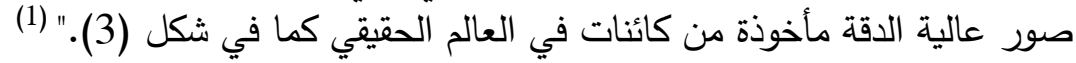

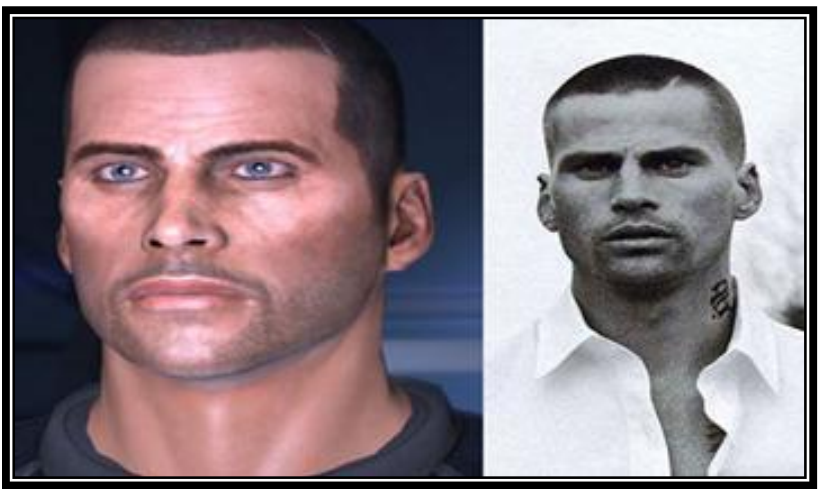

Commander Shepard Mark Vanderloo shepard شكل (3) شخصية حقيقية مقارنة بشخصية شييرد

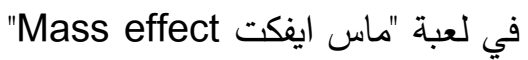

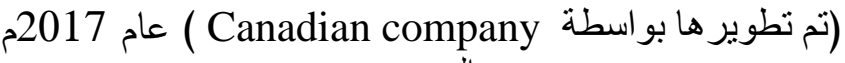
المصدر:

https://callium1456.wordpress.com/2012/12/13/artisticstyles-used-in-video-games/

$$
\text { تأثير الفن الرقمي على جماليات الألعاب }
$$

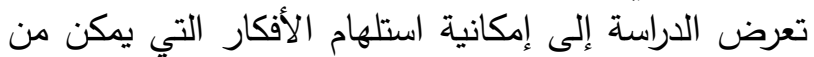

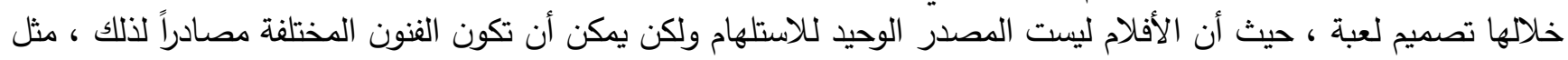

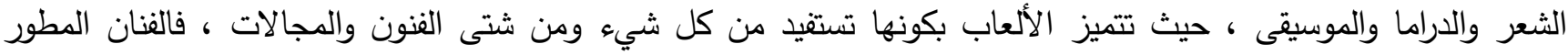

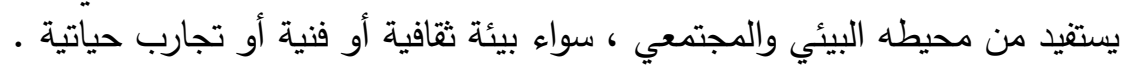

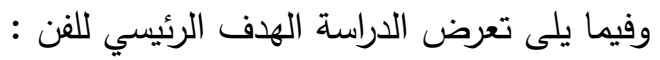

(1) Callium1456 “Artistic Styles Used In Video Games” https://callium1456.wordpress.com/2012/12/13/artistic-styles-usedin-video-games/, 17/12/2018. 


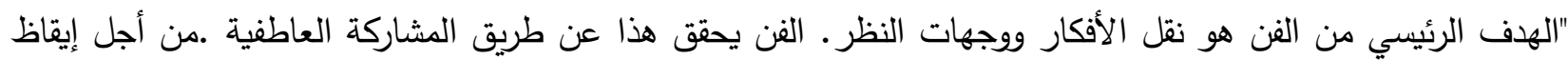

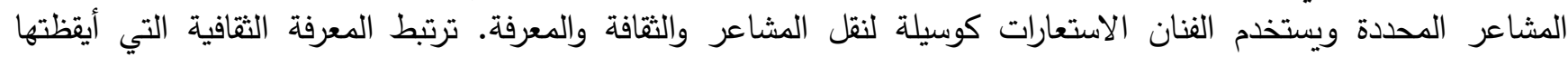
الاستعارات بمشاعر معينة يختارها الفنان. على العكس من ذلك ، يمكن للعواطف إبراز الصور الذهنية والمعرفة والاستعارات مما

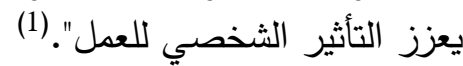
أي إنه على مدى السنوات الماضية تطورت ألعاب الفيديو بصورة واضحة وبشكل متزايد مما كان لله من الأثر على الحياة

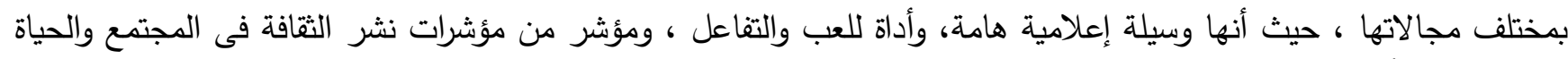
المحيطة ، والألعاب الإلكترونية جاذبة بطبيعة الحال وذلك لما تحويه من جماليات.

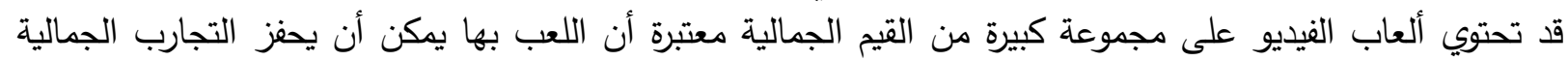

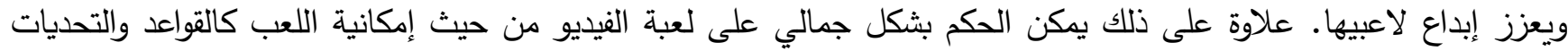

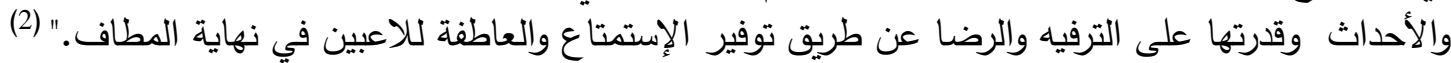

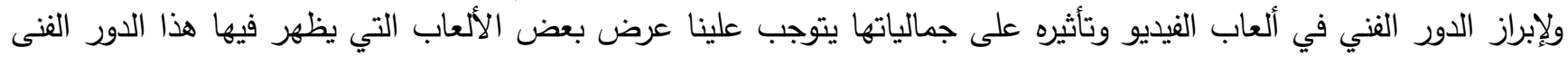

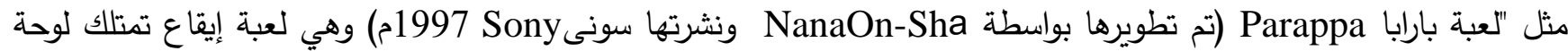

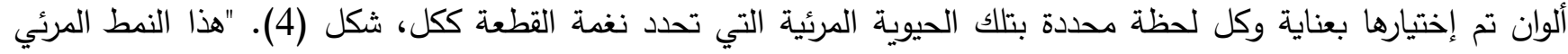

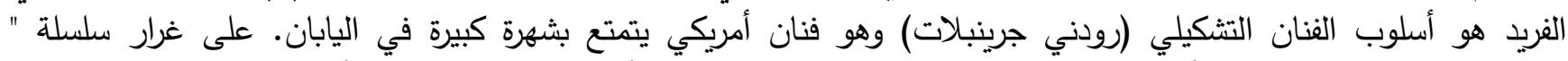

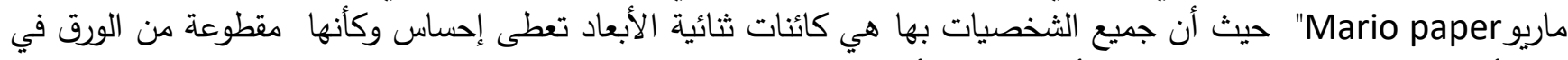

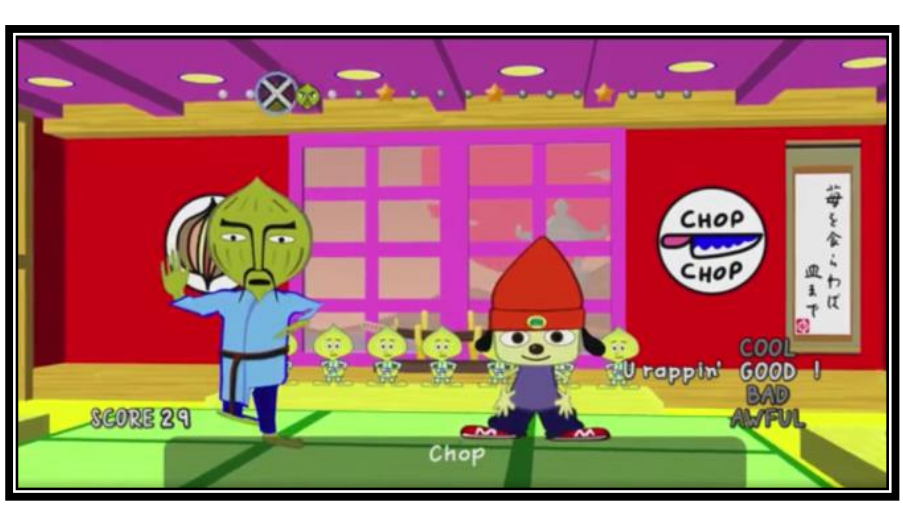
حين أن الخلفيات تكون في المقام الأول ثلاثية الأبعاد."

"وكذلك لعبة الخيال التي تم إنتاجها عام 2006م "النسيان" The Elder Scrolls: Oblivion "هي لعبة أدوار تم تطويرها بواسطة Bethesda Game Studios ونشرت بواسطة Bethesda Softworks تضع التلاعب (في هيئة شخصية اللعبة)

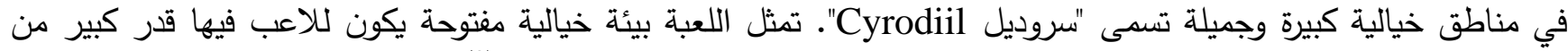

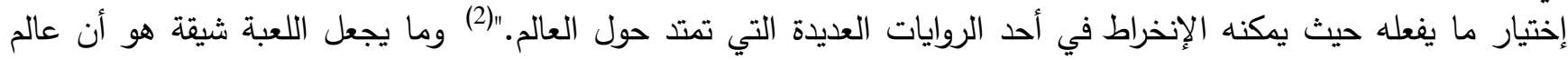

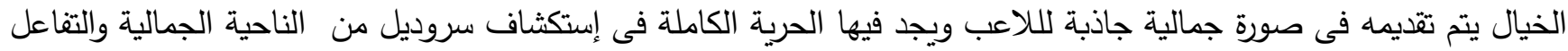

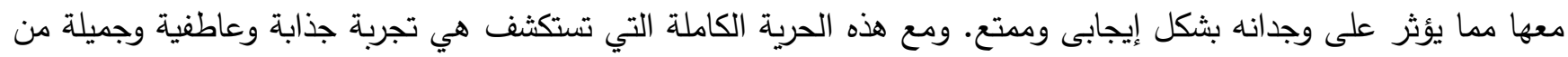

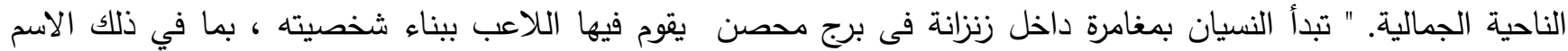

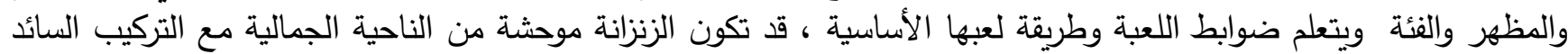

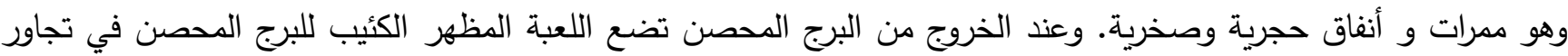

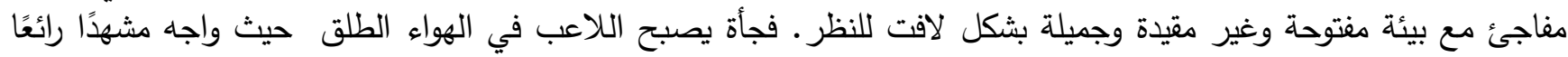

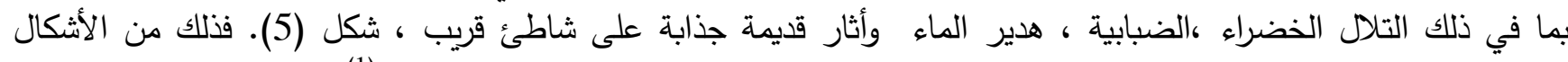
الجديدة في الفن: تمثيل اللاعب وخبراته الجمالية في عالم خيالي يوفر جماليات إستكثافية نشطة".

${ }^{(1)}$ Kevin Bachus "Interview | Kevin Bachus" The art of video games - from Pac-Man to Mass Effect" Welcome Books.

NewYork 2012, p. 66.

(2) di Nuno Fonseca "Hitting the "play" button: the aesthetic values of videogame experience" Itinera, N. 11, $2016 . \mathrm{p} 88$.

(1) Wikipedia “PaRappa the Rapper” https://en.wikipedia.org/wiki/PaRappa_the_Rapper, 15/1/2018.

(2) Wikipedia "The Elder Scrolls IV: Oblivion" https://en.wikipedia.org/wiki/The_Elder_Scrolls_IV: _Oblivion, $1 / 12 / 2018$.

(1) Grant Tavinor "The Art of Videogames", Wiley-Blackwell, 2009, p. 2, 3. 


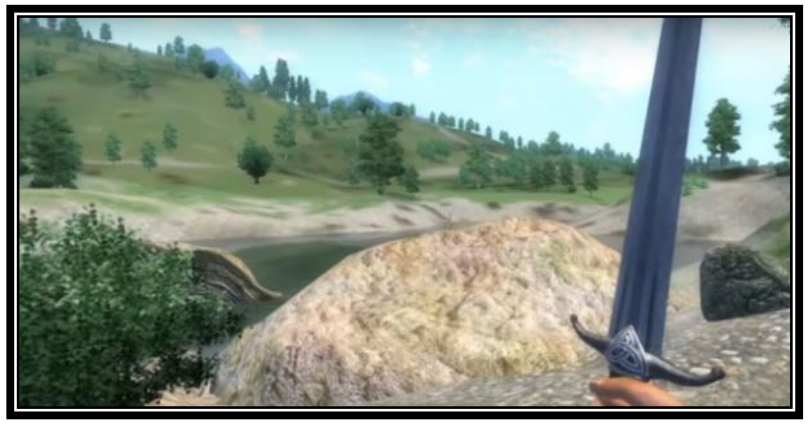

البيئة المفتوحة

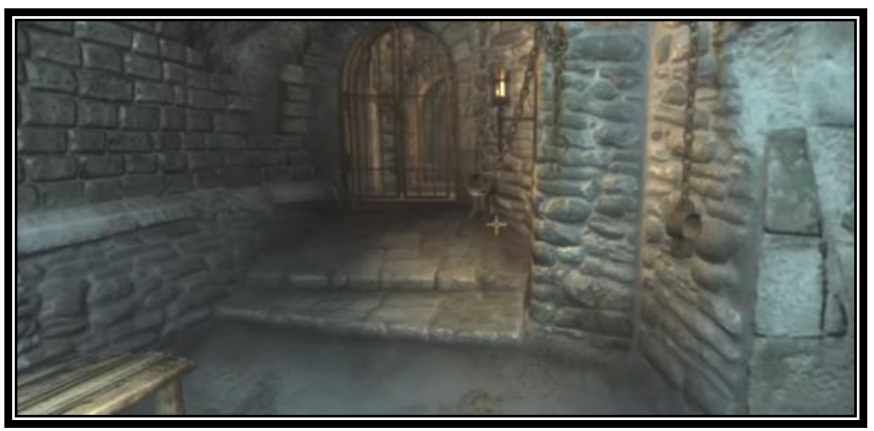

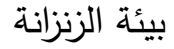

\section{6 (Oblivion) شكل (5) كادرين من لعبة النسيان}

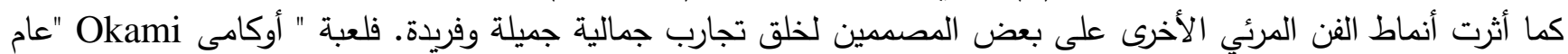

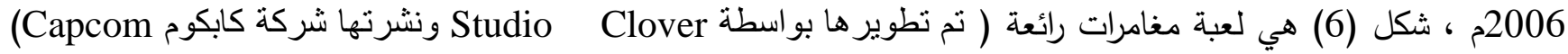

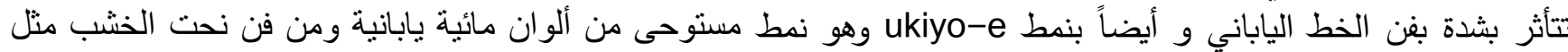

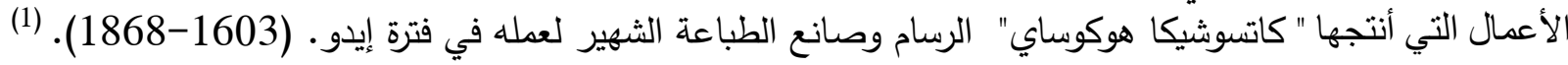
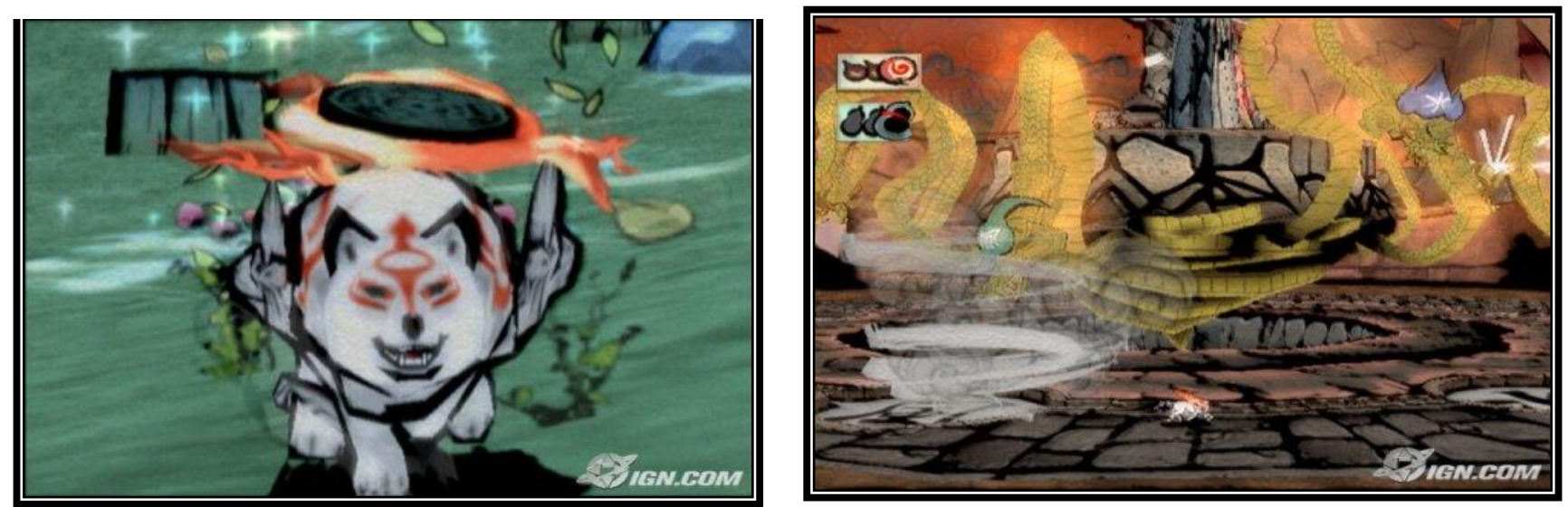

$$
\text { شكل (6) كادرين من لعبة "اوكامى Okami" عام 2006م }
$$

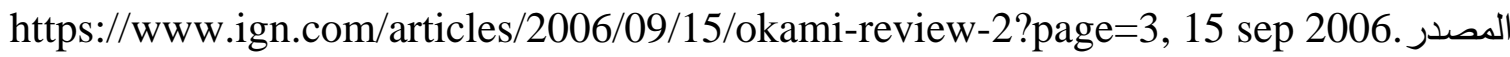

يتم اليوم إنثاء ألعاب الفيديو بمثل هذه التفاصيل الواقعية التي تظهر في بعض الأحيان وكأنها فيلم بحد ذاته. ويمكن رؤية ذلك في لعبة أنشارتد Uncharted 4 حيث تبدو حركات الجسم وتعبيرات الوجه والبيئة الجغرافية جذابة للغاية كما في شكل (7). هذانه
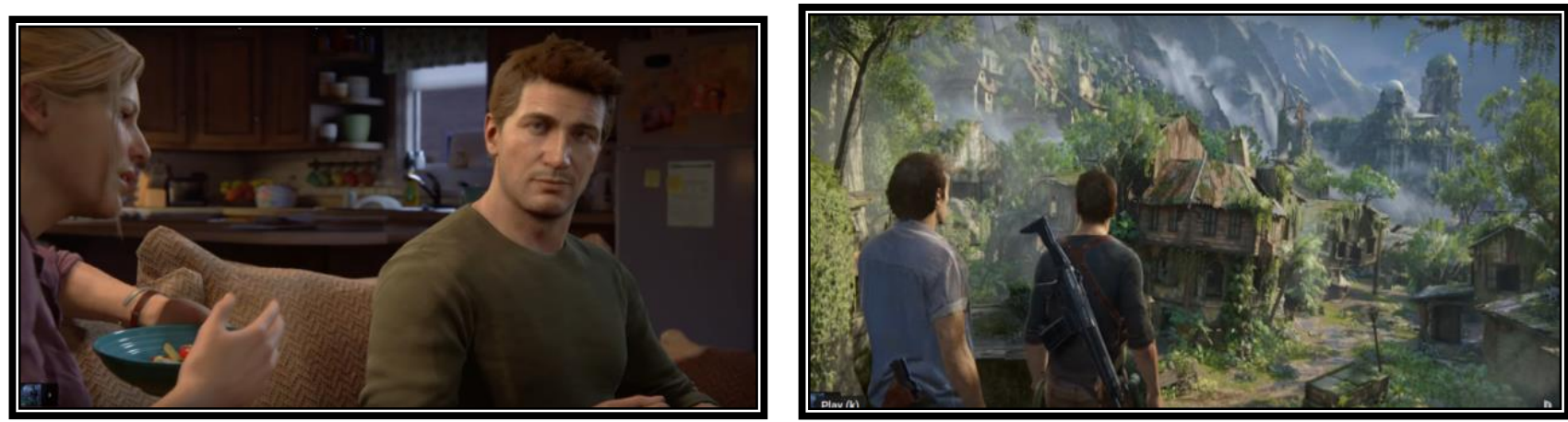

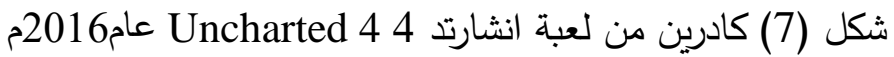

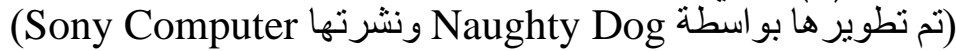

(1) Sam Jones"Okami HD "Traditional art graphics in a modern game" https://www.fanatical.com /en/blog/okami-hdtraditional-art-graphics-in-a-modern-game, 5/11/2018. 
الأيدولوجية تثبه إلى حد كبير الفن اليوناني. تجعل جماليات المشاهدة في لعبة أنثارتد4 اللاعب يقدر البيئة ويستمتع باللعبة

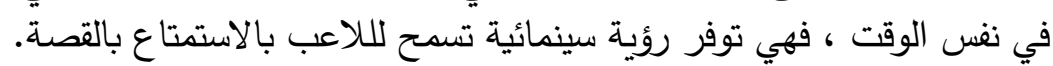

بينما تهدف ألعاب الفيديو الحديثة الأخرى إلى منح المستخدم تجربة واقعية ، فإن لعبة (ليمبو Limbo)، شكل (8) تقوم

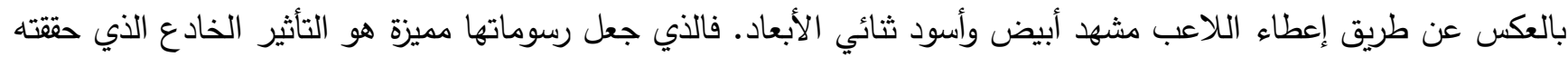

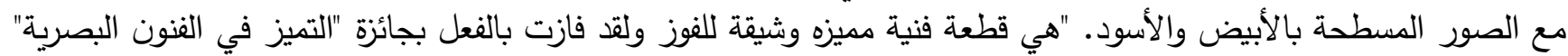

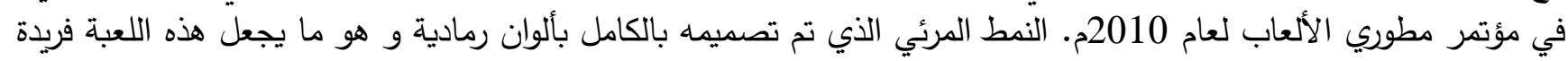

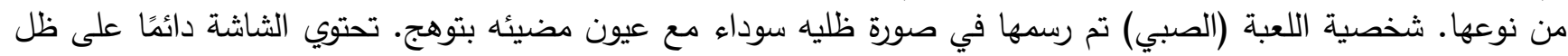

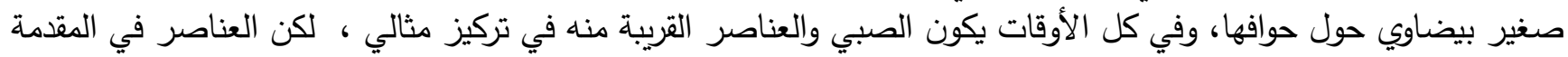

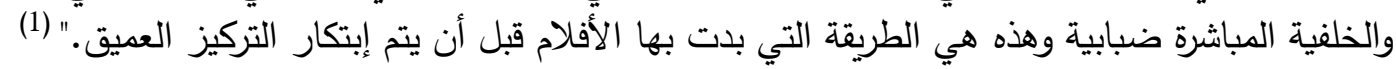
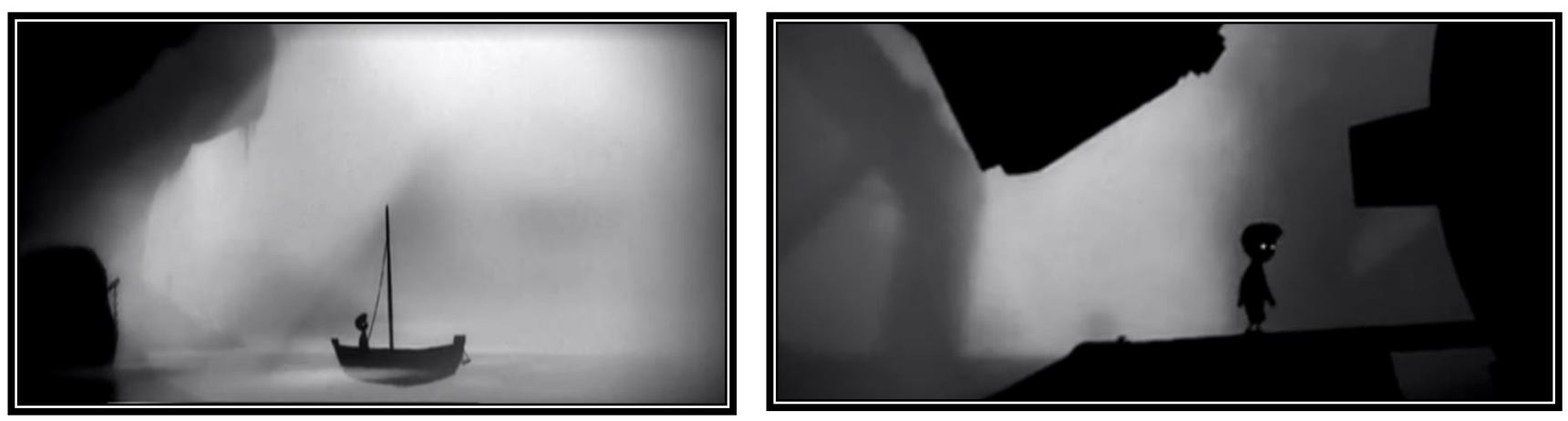

شكل (8) كادرين من لعبة (2010) Limbo)

(

واحدة من أكثر الألعاب الهادئة هي لعبة " وادي النصب التذكاري Monument Valley ". "ت "تطلق هذه اللعبة من قطعة فنية

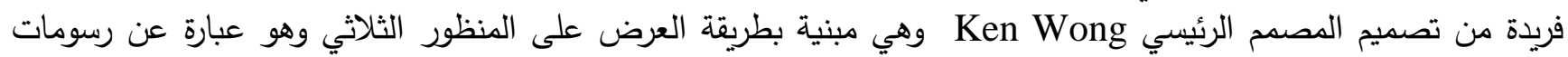

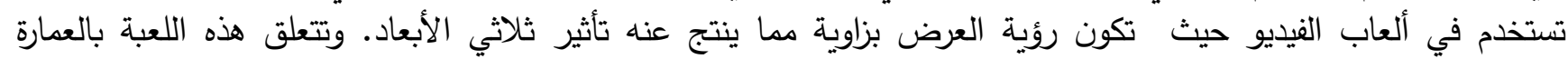

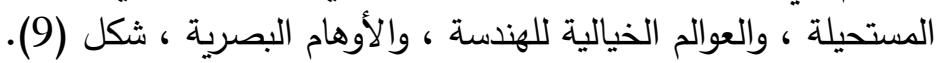

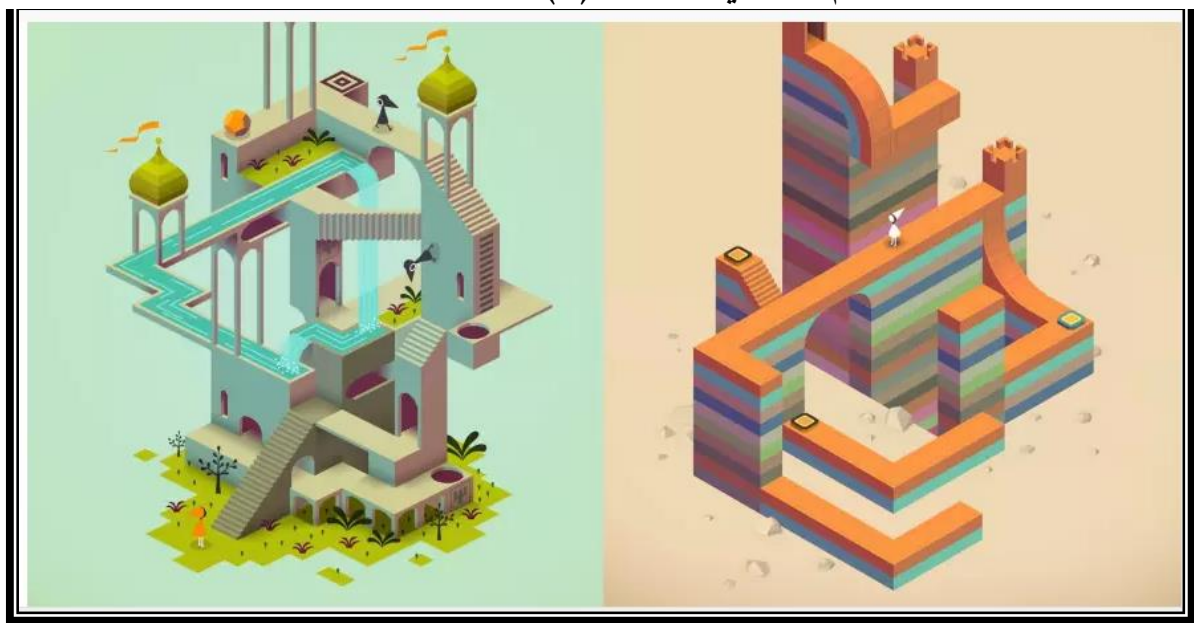

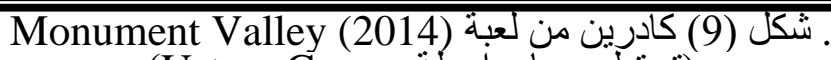

(Ustwo Games تم تطوير ها بو أسطة

https://www.format.com/magazine/galleries/design/video-game-designs.: المصويرن

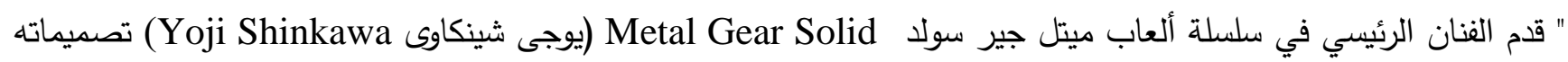
منذ اللعبة الأولى في شكل رسومات قلم رصاص أنيقة معبرة ثم تم تصميمها في اللعبة بواسطة فريق من الفنانين الرقميين في

(1) Review-Journal "Visual style makes 'Limbo' a unique game " August 7, 2010

https://www.reviewjournal.com/life/visual-style-makes-limbo-a-unique-game/, 9/12/2018. 
( هideo Kojima هالتصميم الميكانيكي هو الشيء الذي تفوق فيه فإن الدبابة ثنائية البرمائيات (راى Ray )

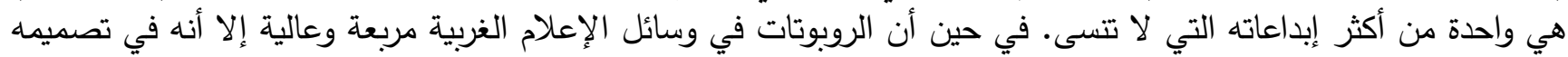
فضل إبراز المزيد من التصميمات العضوية. فبالرغم من أن (راى Ray ) عبارة عن آلة ، ولكنها تبدو وكأنها مخلوق بحري ،

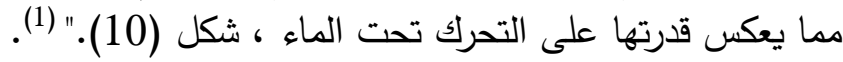

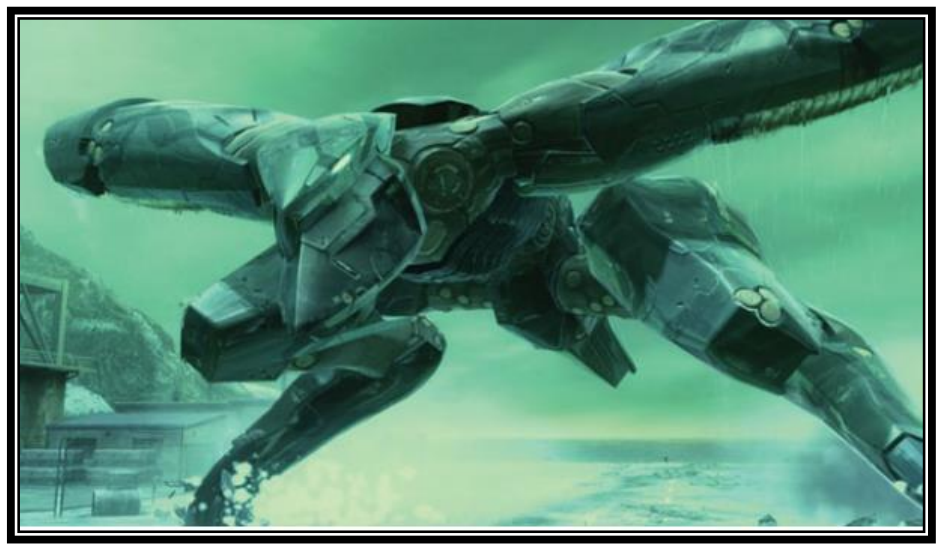

Metal gear RAY شكل (10) كادرين من لعبة (2014) (2014)

(تم تطوير ها بو اسطة (Ustwo Games) المصدر:

https://www.format.com/magazine/galleries/design/video-game-designs

\section{العلاقة بين الفن وألعاب الفيديو}

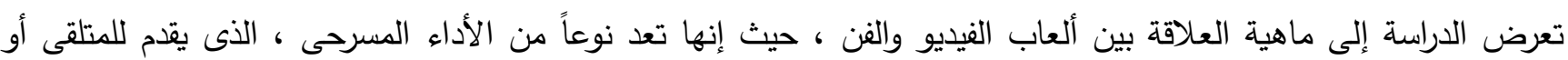

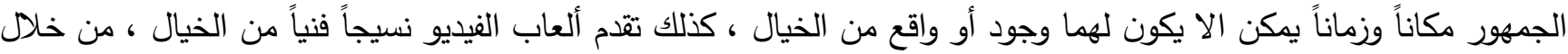

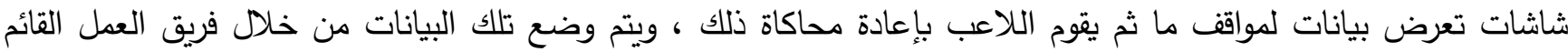

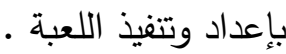
تعود العلاقة بين الفن وألعاب الفيديو إلى بداية تاريخ ألعاب الفيديو تقريبًا. فقد شاركوا المبرمجين والمصممين الأوائل

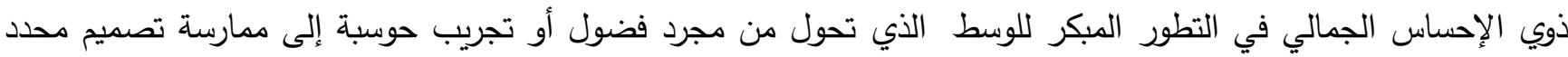

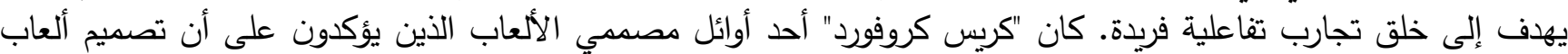

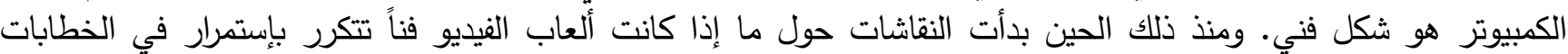

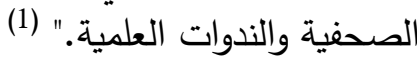
" في بداية الثمانينات إنفجرت سوق ألعاب الفيديو والحواسيب الثخصية. فكانت شركات مثل أتاري وماتل وكوليكو وكومودور

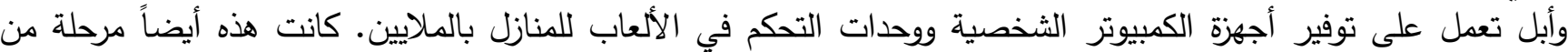

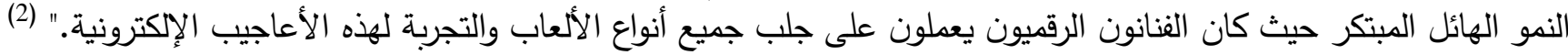

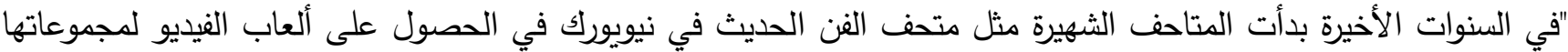

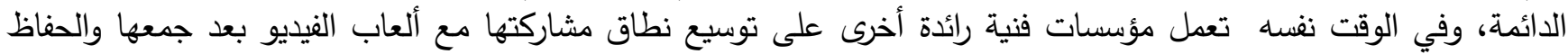

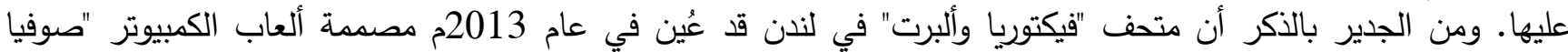

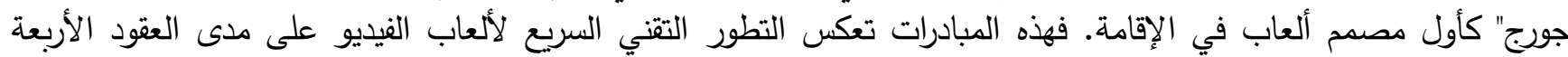

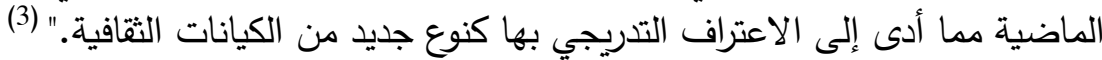
ونخلص إلى أن ألعاب الفيديو في إطارها الرقمي الجديد تحقق العديد من الأهداف والوظائف التي تربطها بالفن تاريخياً وثقافياً

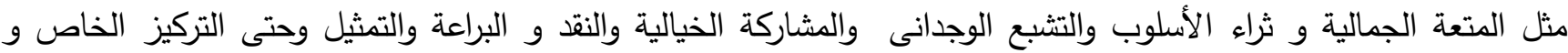

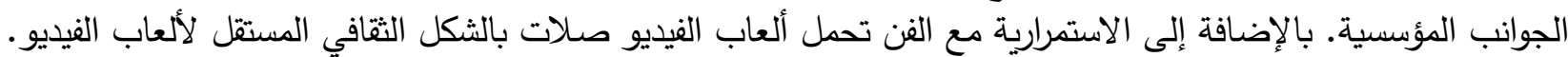

(1) Andy Kelly "20 best designs in video games" https://www.creativebloq.com/inspiration/20-best-designs-in-videogames-1233904/, 9/12/2019.

(1) Mark J.P. Wolf "Encyclopedia of video games : the culture, technology, and art of gaming", Greenwood, California, USA, 2012., p. 38.

(2) Chris Melissinos and Patrick O'Rourke "The art of video games - from Pac-Man to Mass Effect", Welcome Books. New York 2012, p. 15.

(3) Bill Balaskas "In and Out of Art History: The Video Games Conundrum" published: Mar 21, 2017, https://arthist.net/archive/15011, 15/11/2018. 


$$
\begin{aligned}
& \text { - - الفن الرقمي أزاد الإدراك الحسي والفهم للفن وتغطية أحداثه. }
\end{aligned}
$$

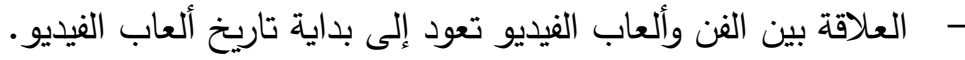

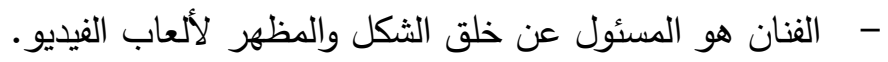

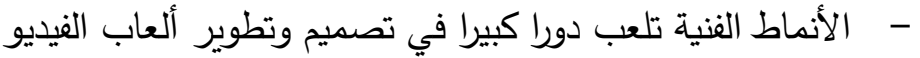

$$
\begin{aligned}
& \text { - - الفن الرقمي أعطى للمصممين الفرصة لخلق تجارب جمالية فريدة. } \\
& \text { التوصيات : - الت } \\
& \text { 2- ضرورة تعمق الباحثين أكثر في دراسة الفن الرقمي والأنماط الرسومية في الألعاب الفئ الفيديو. } \\
& \text { 3- يجب على الفنانين الإهتمام بتعلم الفن الرقمي وأدواته الرقمية . }
\end{aligned}
$$

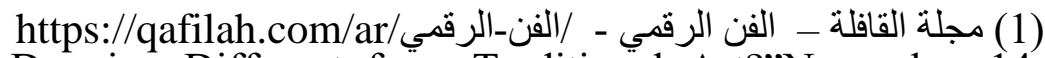
(2) Autodesk, Inc. "How Is Digital Drawing Different from Traditional Art?"November 14, 2017https://www.sketchbook.com/blog/digital-drawing-different-traditional-art/.

(3) Enrico Nardelli "A classification framework for interactive digital artworks" International Conference on User Centric Media UCMEDIA 2010.

(4) Lee, L., Gee, S. \& Dolah, J. "Graphic Styles Appearance in Educa-tional Games to Enhance Malaysian Students Learning: A Preliminary Study". $2^{\text {nd }}$ international conference on creative media, design \& technology (REKA2016).

(5) Callium1456 "Artistic Styles Used In Video Games" https://callium1456.wordpress.com/2012/12/13/artistic-styles-used-in-video-games/.

(6)Kevin Bachus "Interview | Kevin Bachus" The art of video games - from Pac-Man to Mass Effect" Welcome Books. NewYork 2012.

(7) di Nuno Fonseca "Hitting the "play" button: the aesthetic values of videogame experience" Itinera, N. 11, 2016.

(8) Wikipedia "PaRappa the Rapper"https://en.wikipedia.org/wiki/PaRappa the_Rapper.

(9) Wikipedia "The Elder Scrolls IV: Oblivion" https://en.wikipedia.org/wiki/The_Elder_Scrolls_IV:_Oblivion.

(10) Grant Tavinor "The Art of Videogames", Wiley-Blackwell, 2009.

(11) Sam Jones"Okami HD "Traditional art graphics in a modern game" https://www.fanatical.com /en/blog/okami-hd-traditional-art-graphics-in-a-modern-game.

(12) Review-Journal "Visual style makes 'Limbo' a unique game" August 7, 2010

https://www.reviewjournal.com/life/visual-style-makes-limbo-a-unique-game/.

(13) Andy Kelly "20 best designs in video games" https://www.creativebloq.com/inspiration/20best-designs-in-video-games-1233904/.

(14) Mark J.P. Wolf "Encyclopedia of video games : the culture, technology, and art of gaming", Greenwood, California, USA, 2012.

(15) Chris Melissinos and Patrick O'Rourke "The art of video games - from Pac-Man to Mass Effect", Welcome Books. New York 2012.

(16) Bill Balaskas "In and Out of Art History: The Video Games Conundrum" published: Mar 21, 2017, https://arthist.net/archive/15011. 